\title{
Servicios bibliotecarios: la perspectiva de los estudiantes con discapacidad y el personal bibliotecario a través de seis universidades madrileñas
}

\author{
Ana Nieves Millán Reyes*
}

Resumen: Se presentan los principales resultados de la investigación sobre las Bibliotecas Universitarias de la Comunidad de Madrid, en relación con los usuarios con discapacidad y su personal, junto con la percepción sobre el servicio prestado. La metodología emplea el cuestionario como instrumento de medida para extraer los datos, resultados y conclusiones de ambos colectivos. El análisis estadístico ha sido descriptivo y bivariable (pruebas no paramétricas). Los resultados principales indican la existencia de una población de usuarios con alguna discapacidad, sus hábitos, y su percepción sobre los fondos, infraestructuras y materiales bibliotecarios. Existen servicios destinados a estudiantes con discapacidad, pero no están extendidos por toda la geografía universitaria y tampoco comparten criterios. Se pone de manifiesto la necesidad de fomentar la sensibilización del personal bibliotecario, además de mejorar la organización de las bibliotecas.

Palabras clave: Bibliotecas universitarias, discapacidad, personas con discapacidad, servicios bibliotecarios, legislación, Comunidad de Madrid, servicios especiales, accesibilidad, diversidad funcional, personal bibliotecario.

\section{Librarian services: a perspective of students with disabilities and the library staff of six university libraries in Madrid}

\begin{abstract}
Main results about the research on the University Libraries in the Region of Madrid in connection with users with disabilities and their staff are shown. Also the perception of the service seen by both groups. The methodology uses the questionnaire as a measuring instrument to extract the data, findings and conclusions of both groups. Statistical analysis was descriptive and bivariate (non-parametric tests). The main results are the finding of a population of users with disabilities, and its habits, perceptions about the funds, facilities and library materials. The presence of services for students with disabilities are not widespread throughout the universities and don't present common criteria. The need to raise awareness of the library staff is evident, moreover to improve the libraries organitation.
\end{abstract}

Keywords: University libraries, disability, persons with disability, library services, legislation, Madrid Region, special services, accessibility, functional diversity, staff librarian.

* Biblioteca Digital del Voluntariado y las Asociaciones. Liga Giennense para la Educación y la Cultura Popular. Jaén. Correo-e: biva1@biva.es, anmillan@ugr.es.

Recibido: 2-3-09; $2 .^{\text {a }}$ versión: 23-9-09; aceptado: 28-9-09. 


\section{Introducción}

En todas las culturas han existido diferentes discapacidades, cuyo trato y acercamiento ha ido variando a lo largo de la historia. Así, las deficiencias, discapacidades y minusvalías han generado diferentes actitudes y modelos que oscilan, entre la actitud pasiva (rechazo, segregación) o activa (integración, tratamiento), y en las distintas etapas históricas (prehistoria, antigüedad clásica, etc.,). Esta evolución afecta también a los modelos teóricos, que varían de un modelo médico curativo, que pone el acento en el ajuste social y personal para facilitar su adaptación a un modelo social de la discapacidad, en el que se acentúa la igualdad de oportunidades (la sensibilización social, el diseño universal, la inclusión social, etc.) la cual se extiende hasta el entorno bibliotecario.

Partiendo de las premisas anteriores, se une la función socializadora que tiene y desempeña la biblioteca; recordemos que el hombre es considerado un ser social que necesita relacionarse "principio de socialización", con distintos agentes (personas, iguales o pares, familia, amigos, instituciones, etc.) lo que nos permite considerar a nuestras instituciones como agentes socializadores, pues cumplen con varias de las dimensiones que componen el proceso socializador. La socialización se constituye y nos constituye como un instrumento, no sólo para la transmisión del patrimonio cultural, sino también para la educación, la alfabetización de toda la sociedad. La biblioteca materializa esa función mediante diversas experiencias o iniciativas llevadas a cabo, desde los distintos tipos de centros: el servicio especializado de atención a personas sordas en la Biblioteca Forum Metropolitano, el Servicio de Documentación y Traducción de la ONCE y su sistema bibliotecario y la creación de recursos destinados a personas con discapacidad (PREDISCAN (Pinto, 2008a), CREADIS (Pinto, 2009), GUIADIS, etc.), iniciativas para la alfabetización informacional (Pinto, M., 2008b), como el Blog sobre Alfin y discapacidad, o el portal ALFIN EEES, etc.

No obstante, todo este proceso se relaciona con la evolución normativa (Millán, 2008b), de las últimas décadas, que ha logrado grandes avances en los distintos entornos geográficos en pro de los derechos y deberes de las personas con discapacidad y cuyos principios superiores (principio de derecho, integración, igualdad de oportunidades) se han ido reflejando y plasmando en los distintos entes legislativos como la ONU, la UE (Figura 1), así como los tipos de medidas que se han generado; medidas de acción positiva y de acción contra la discriminación, que se reflejan en la mayor parte de las leyes. Principios que se han traslado también, en mayor o menor grado a la normativa española, (Constitución Española, Ley 51/2003, de igualdad de oportunidades) y al actual sistema universitario, que se encuentra en un momento de cambios, donde la adecuación de sus servicios a las demandas sociales es un aspecto a tener en consideración (Guerrero, 2005) en la que los estudiantes con discapacidad son cada vez más visibles en la vida universitaria.

Los argumentos anteriores de índole legal, social (modelo social de la discapacidad), igualdad de oportunidades, profesionales (calidad de los servicios bi- 
FIGURA 1

Principios Mencionados en la Legislación

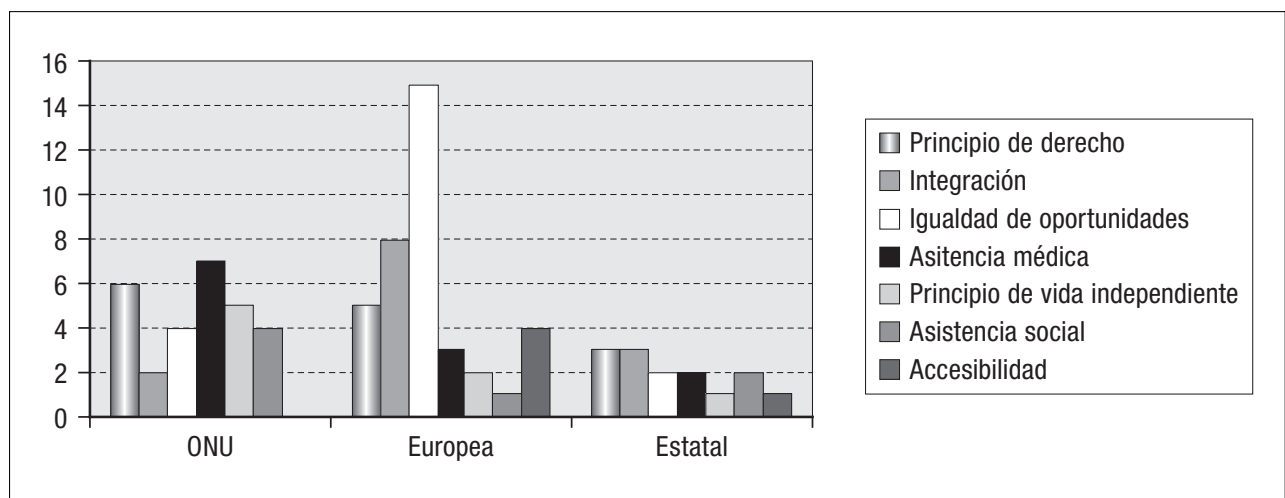

bliotecarios, función socializadora) justifican la investigación sobre los estudiantes con alguna discapacidad, su perfil y la relación con las bibliotecas universitarias, a través de un análisis descriptivo, para poder hacer patente su existencia y así poder mejorar los servicios. Del mismo modo que con el personal bibliotecario del que se desea conocer el perfil, la percepción sobre estos usuarios y su biblioteca.

Los objetivos cardinales son, conocer, por una parte, las actuales bibliotecas universitarias madrileñas están al servicio de estas personas y, de otra parte, la percepción sobre el servicio prestado en la biblioteca por parte del personal a los estudiantes con alguna discapacidad.

Para contextualizar y abordar el objeto de estudio se ha realizado una amplia revisión bibliográfica en distintas bases de datos, vaciándose todos aquellos artículos que estuviesen relacionados con la investigación, el contexto, el desarrollo, el marco, la terminología (Cebrián, 2004) y los recursos para la supresión de barreras de comunicación de las personas sordas (Santos, 2003).

Algunos antecedentes localizados comparten una temática variada y una orientación diversa, encontrándose: estudios sobre las personas con discapacidad física y los equipamientos proporcionados en bibliotecas universitarias del Norte de Carolina (Halle e Esineman, 2005), las personas con discapacidad visual en las bibliotecas públicas (Evans, 2000, Griebel, 2000), los servicios bibliotecarios para las personas con discapacidad (Brazier, 2005; Evans, 2000; Griebel, 2000; Epp, 1999; Millán, 2008; Millán, 2006), para personas ciegas en bibliotecas públicas (Byrne, 2005; Gallo, 2004), iniciativas con diferentes colectivos como sordos, y discapacitados psíquicos (Ortiz, 2003), la creación de centros de interés sobre discapacidad (Teijo, 2003), bibliotecas móviles (Charoenpaed, 2000), en secundaria (Epp,1999) y sobre modelos de trabajo para servir de apoyo a la lectura 
(Centrum för Lättlast, 2000). En relación con la accesibilidad en sus distintas modalidades (Térmens, 2008; Christiensen, 2001; Forrest, 2005; Gutiérrez, 2001; Hassan, 2004), como las revistas electrónicas (Coonin, 2002), páginas web (Coombs, 2000; O’neill, 1998), la edificación del edificio, eliminación de barreras arquitectónicas (Church, 2002; Maldonado,1998); en bibliotecas parlamentarias (Kedem, 2001), acerca de las tecnologías de la información como el telebook, para facilitar al usuario el acceso al catálogo a través de teléfono con una operación rápida, y sencilla (Cohen, 2000). Además de los beneficios de la biblioteca virtual para las personas con discapacidad (Suárez, 1998; Huidobro, 2001; Gutiérrez, 2001; Romero, 1998), internet y su calidad a través de la usabilidad y accesibilidad (Blandford, 2003; Méndez, 2001). Las herramientas y los productos que se pueden adaptar a los usuarios con discapacidad (Coombs, 1999).

En relación con los comportamientos de los usuarios, se encuentran algunos estudios en bibliotecas universitarias en las que se identifican diversos factores, que pueden afectar al comportamiento de las personas con discapacidad visual que buscan información (Beverley, 2007). Otros estudios señalan la importancia del tipo de centro (Carpenter, 1996). Otros estudios apuestan porque las bibliotecas de ciencias y tecnológicas contribuyan al talento científico entre sus usuarios con discapacidad, haciendo accesible el entorno bibliotecario y asegurando, tanto como sea posible, la independencia para el acceso a la información (Coonin, 2001).

\section{La universidad, las bibliotecas universitarias españolas y las personas con discapacidad}

La aproximación del contexto universitario español en relación con los estudiantes con discapacidad, tiene sus comienzos en los años noventa con la puesta en marcha de forma paulatina de los servicios o programas para personas con alguna discapacidad (PIUNE, programa de integración de los universitarios con necesidades especiales de la UAB en el curso 1992-1994, etc.) que después se han ido extendiendo al resto de universidades en mayor o menor medida y que son conocidos como servicios de apoyo, unidades de integración, etc. (Millán, 2008a).

En el marco bibliotecario universitario, el acercamiento a las personas con discapacidad se produce también en esta década, aunque no disponemos de las fechas de creación de los distintos servicios específicos para estudiantes con discapacidad, pero existen publicaciones y autores que tratan la problemática en estos años como Pérez (1998), en la cuál habla de una biblioteca para discapacitados. También se trata en otros entornos, como las bibliotecas públicas sobre servicios especiales (Mayol, 1994), o sobre fuentes documentales (Marqués, 1996) para personas con discapacidad, pero no se encuentran publicaciones relativas a las esferas de las bibliotecas universitarias.

Se puede decir que los comienzos de estos servicios específicos de la biblioteca universitaria se traducen en la prestación de determinadas ayudas técnicas, 
pero que, poco a poco, han ido evolucionando hacia una tendencia más inclusiva, fruto de nuevas formas de entender la biblioteca universitaria, basadas en un diseño para todos y con espacios abiertos como los CRAIs. Algunos de estos servicios y sus características pueden verse en la página web del propio servicio bibliotecario (Universidad Carlos III, Universidad Complutense, Universidad de Granada, etc.) con distintas denominaciones (servicios para estudiantes con discapacidad, etc.) fruto del entendimiento de la problemática por parte de las distintas instituciones.

En un primer momento no existe un modelo consensuado en cuanto, a los elementos que han de componer estos servicios (Millán, 2006). A posteriori se han incorporado algunos nuevos, como ocurre con el prestado por la Universidad de Alcalá (emprendido en el año 2007), cuya biblioteca en el momento del inicio de este estudio no disponía de servicio específico y que en la actualidad se encuentra bastante desarrollado (Saldaña, 2008).

\section{Metodología}

La metodología ha combinado los métodos de investigación documental y el analítico. A través del primero se han recopilado y estudiado las fuentes de información necesarias para la realización de la investigación, el segundo se ha utilizado para el análisis y valoración de la información recopilada. La estrategia de investigación se ha basado en la encuesta, empleando el cuestionario como instrumento de recogida de datos.

El ámbito se desarrolla en la Comunidad de Madrid, curso académico 2004/2005 y 2005/2006. Los destinatarios han sido los usuarios con alguna discapacidad y el personal bibliotecario de las seis universidades madrileñas seleccionadas. La hipótesis de partida plantea que en el ámbito de la Comunidad de Madrid, la realidad de los servicios de las bibliotecas universitarias no se relaciona con las características de las personas con discapacidad o diversidad funcional. La otra hipótesis planteada propone que en el ámbito de la Comunidad de Madrid la realidad de los servicios de las bibliotecas universitarias difiere entre personas con discapacidad y el personal bibliotecario. De acuerdo con las hipótesis tenemos las siguientes variables para ambas hipótesis:

- La variable independiente: características de las personas con discapacidad que comprenden el género, el tipo de discapacidad, la titulación, el curso, la universidad, etc.

- La variable independiente: características del personal bibliotecario universitario madrileño que abarcan el género, la escala profesional, la universidad dónde trabaja, la biblioteca dónde trabaja.

- Como variable dependiente: la realidad de los servicios de las bibliotecas universitarias que incluye entre otras, el uso de los servicios, la frecuencia de uso, el tipo de servicio, la señalización, la adecuación de las instalaciones, la formación del personal, etc. 
El Diseño se corresponde con un diseño descriptivo y de carácter seccional (curso académico 2004/2005 y principios del 2005/2006). Las muestras de la investigación se formaron por: la muestra de usuarios y la muestra de personal. En cuanto al error muestral hemos de aclarar que al no haber sido realizado un muestreo probabilístico, éste no puede ser calculado. Es sólo estimable en muestras probabilísticas. El instrumental (cuestionario) empleado, estaba estructurado en preguntas cerradas, abiertas, y en un orden definido. El número total de las preguntas es de 27, para el cuestionario destinado a estudiantes con discapacidad y de 22, para el personal bibliotecario de las universidades. La naturaleza de las preguntas recoge facetas de identificación (el género, el tipo de discapacidad, la escala profesional, etc.), de acción (uso de los servicios bibliotecarios, etc.), de opinión (sobre las instalaciones, etc.), de información (para comprobar los conocimientos de los encuestados, sobre disponibilidad de tecnologías de asistencia, o los servicios de apoyo a estudiantes con discapacidad, etc.), de intención (interés en los cursos de formación, etc.), de motivación (razones por las que va a la biblioteca, etc.)

El procedimiento comenzó con un entrenamiento personal (pautas sobre la localización de los entrevistados, los procedimientos de motivación para la participación, la comprobación de las respuestas, etc.), a continuación un estudio piloto o pretest del cuestionario; la revisión de éste fue realizada por un grupo de expertos de forma anónima que señaló las mejoras sobre la redacción, la forma de las preguntas, las respuestas y el orden de los temas. Tras ésta, se realizó la modificación del cuestionario para pasar a su posterior aplicación en el segundo semestre del curso académico 2004/2005 y principios de 2005/2006. El trabajo de campo consistió en la visita a las distintas facultades y escuelas de las universidades seleccionadas en la Comunidad de Madrid, para encuestar tanto a los estudiantes con alguna discapacidad, como al personal bibliotecario. Se combinaron diversos métodos para la cumplimentación de ambos cuestionarios: la encuesta personal, la telefónica, por correo electrónico y autoadministrada para los estudiantes con discapacidad. Para el personal bibliotecario, se utilizó la encuesta personal, la autoadministrada y por correo electrónico con la colaboración del personal facultativo (UAM, URJC), que distribuyó internamente el cuestionario entre el personal dentro de los plazos establecidos. Se realizó un seguimiento y control del trabajo tras la recogida de los cuestionarios, a fin de detectar algún error formal o conceptual.

Previo al tratamiento estadístico con las herramientas estadísticas SPSS (14) y la hoja de cálculo excel, se realizó la clasificación de las respuestas, para su posterior grabación. El análisis de los cuestionarios comprende un análisis descriptivo (univariable y bivariable), un análisis inferencial con la aplicación de pruebas no paramétricas, para saber en que grado están asociadas las variables, mediante las tablas de contingencia, las pruebas del chi-cuadrado para variables cualitativas y la prueba t-de student, para variables con nivel de medición de intervalo o de razón. Para poder llevar el análisis de los datos y la realización de las pruebas se procedió a la unificación de aquellas categorías o modalidades que 
eran demasiado amplias, de modo que se pudiera cumplir con la condición de validez, para la realización de dichas pruebas.

\section{- Universo y población}

Para determinar la población se contó con la base de la muestra o marco muestral (listas que comprenden las unidades muestrales de la población) para ambos colectivos (estudiantes con discapacidad y personal bibliotecario).

La elección de las universidades madrileñas se basó en el criterio relativo a la posesión de servicios relacionados con las personas con discapacidad. Atendiendo a éste, se extrajeron del Registro Nacional de Universidades y Centros de enseñanza del Ministerio de Educación y Cultura, seis universidades públicas y privadas (UCM, UC3M, URJC, UPM, UPCO, UAM). El marco muestral del personal se formó a partir del directorio disponible en las universidades madrileñas correspondiente al curso académico 2005/2006, momento en que se finalizaba la recogida del cuestionario con un total de 808 sujetos, distribuidos entre las seis universidades (Tabla I).

Para determinar la población de estudiantes con discapacidad, nos dirigimos a las oficinas de atención a estudiantes con alguna discapacidad, a las que se realizó la petición de datos durante el primer semestre del curso académico 2004/2005. Como se puede ver en la Tabla I, había un total de 448 estudiantes distribuidos entre las seis universidades.

\section{TABLA I}

Población de estudio del personal bibliotecario y de personas con discapacidad que cursan estudios en el curso académico 2004/2005 y principios del curso académico 2005/2006

\begin{tabular}{c|c|c|c|c|c|c|c}
\hline \multicolumn{1}{c|}{ Universidad } & UCM & UC3M & URJ & UPM & UPC & UAM & Total \\
\hline Personal bibliotecario* $^{*}$ & 371 & 80 & 45 & 170 & 37 & 105 & 808 \\
\hline Personal con discapacidad** $^{*}$ & 213 & 42 & 42 & 49 & 22 & 80 & 448 \\
\hline
\end{tabular}

* Censo disponible curso académico 2005/2006.

** Curso académico 2003/2004. Censo disponible en el curso académico 2004/2005.

\section{- Muestra: elección y tamaño}

Se realizó el muestreo bola de nieve (no probabilístico intencionado) hasta llegar a una saturación teórica. Los factores que han podido influir en el tamaño han sido el tiempo y los recursos disponibles (económicos, materiales, y humanos) la modalidad de muestreo, la diversidad de análisis previstos, la varianza y la heterogeneidad poblacional, junto con el margen de error máximo admisible. 


\section{- Descripción de las muestras (usuarios y personal)}

La muestra de estudiantes con discapacidad se generó a partir de la población de estudio de unos 448 estudiantes, entre las seis universidades, quedando al final constituida por 110 sujetos, que describimos a partir de las siguientes variables, el género, el tipo de discapacidad, la universidad, la titulación, el curso.

Los datos obtenidos de los usuarios con alguna discapacidad (Figuras 2, 3, 4 y 5) en relación con el género nos permiten observar un predominio de los hombres $(53,64 \%)$ sobre las mujeres $(46,36 \%)$. El tipo de discapacidad más frecuente entre los usuarios encuestados es la visual, 59,9\%, en segundo lugar, la

FIGURA 2

Porcentaje de sujetos por sexo

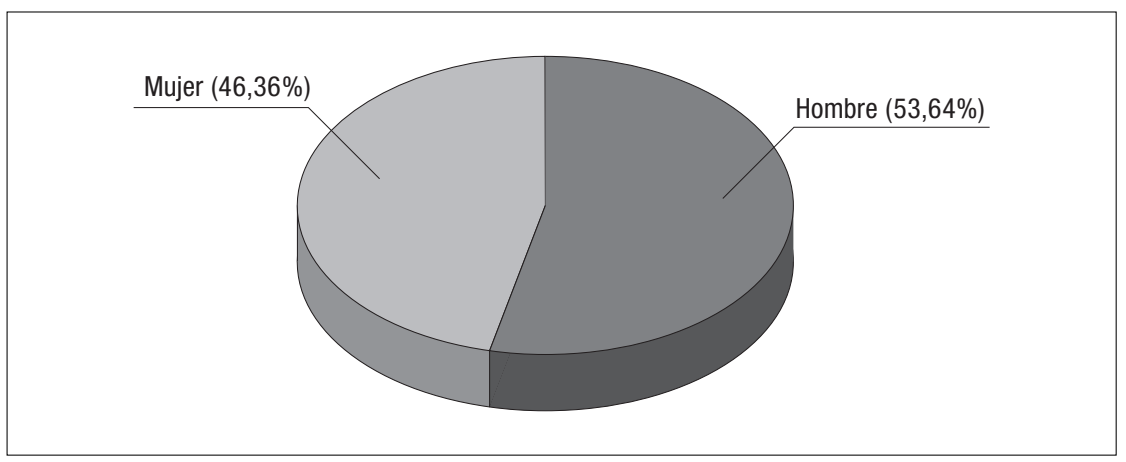

FIGURA 3

Porcentaje del tipo de discapacidad reflejada

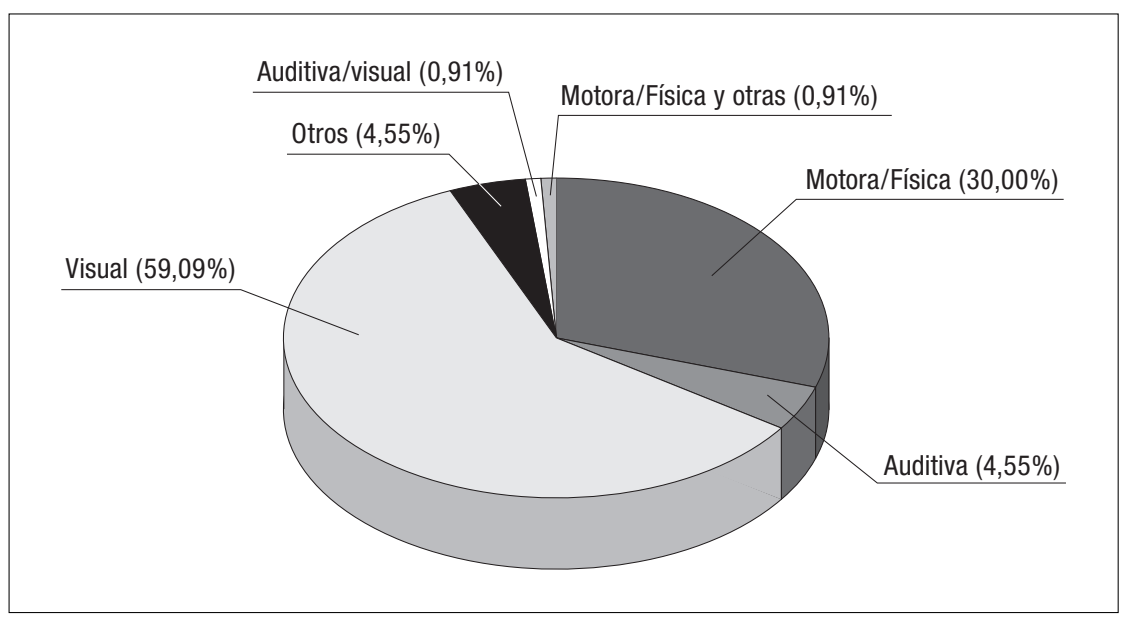


FIGURA 4

Porcentaje de alumnos encuestados por universidad

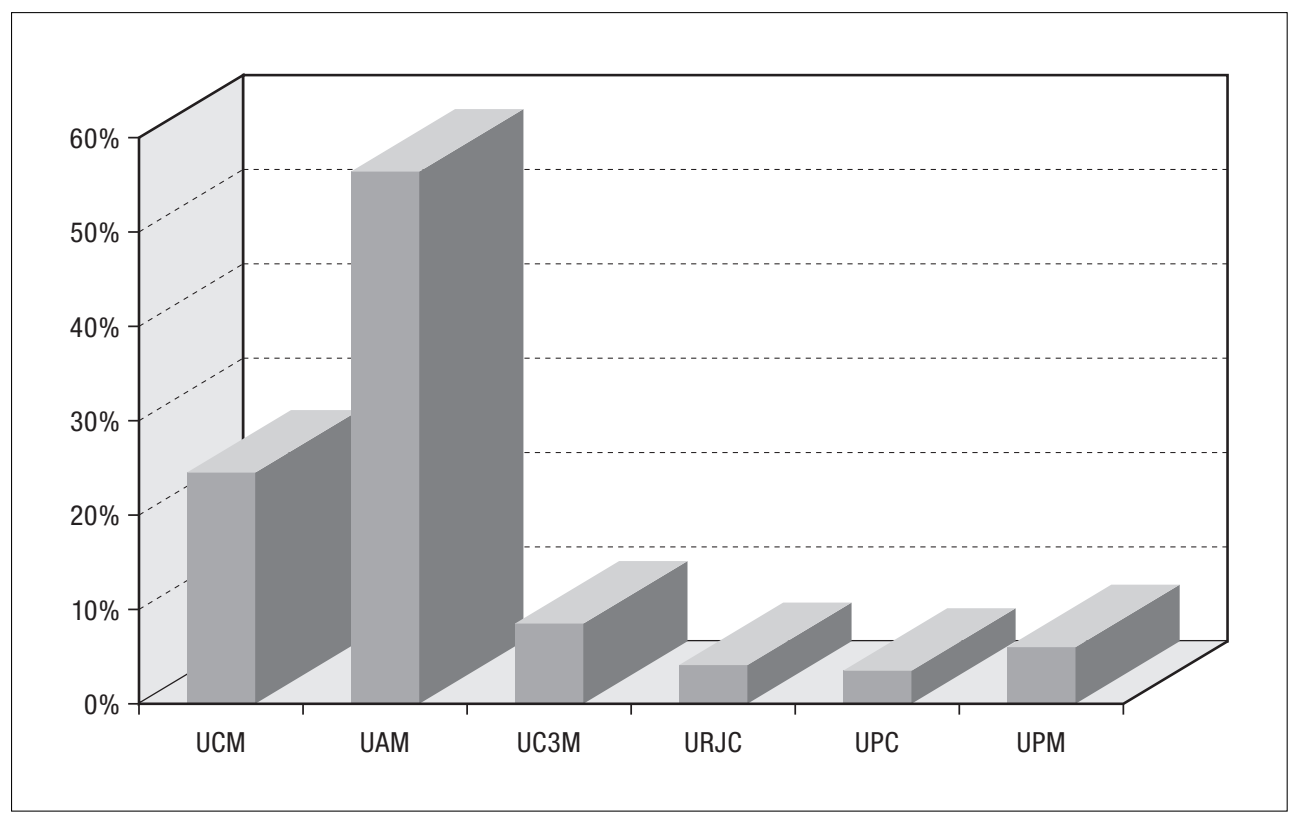

FIGURA 5

Distribución por porcentaje del curso de los encuestados

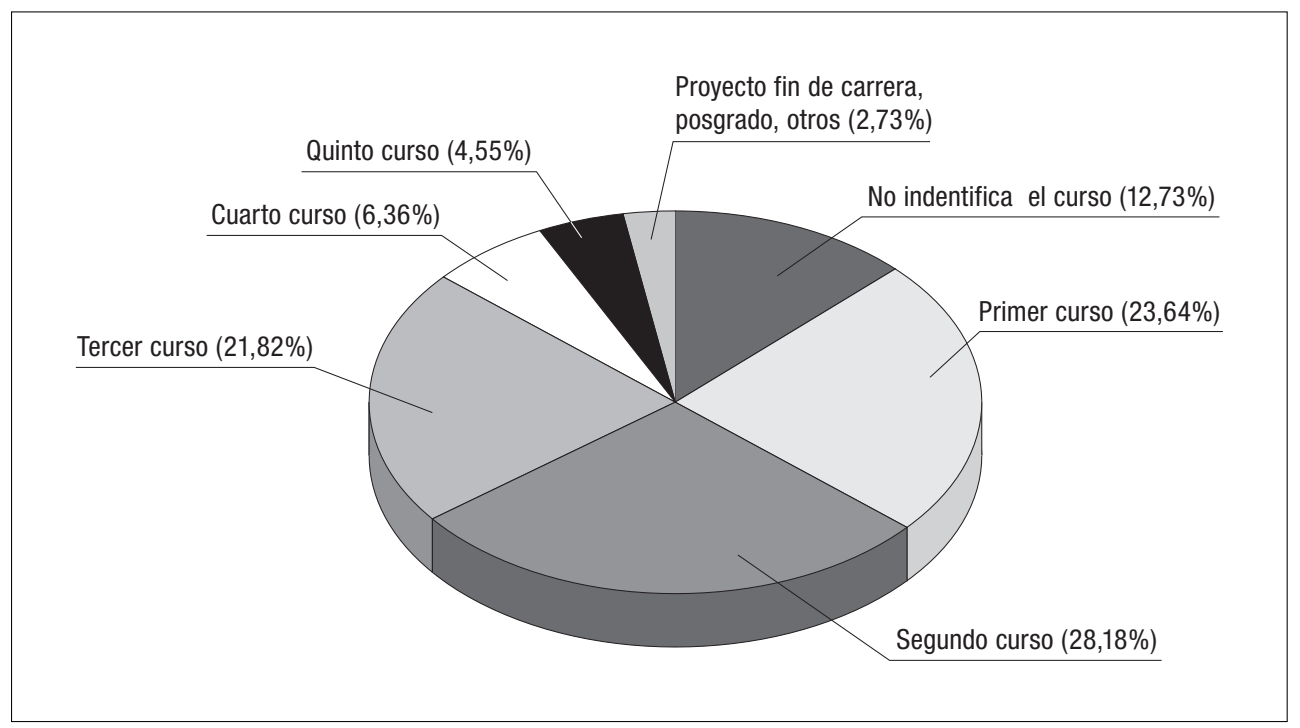


motora/física con un 30,0\%, y en tercero, la auditiva 4,55\%. Las universidades en las que encontramos con más frecuencia usuarios con alguna discapacidad son la Universidad Autónoma (56,4\%) y la Universidad Complutense de Madrid $(24,5 \%)$, seguidas del resto, en menor medida. Las titulaciones más frecuentes cursadas por los estudiantes encuestados son, periodismo, ingeniería informática, psicopedagogía, fisioterapia. La relación entre titulación y género nos indica que hay más presencia del masculino, salvo en algunas titulaciones como enfermería, educación primaria, psicología, etc., donde hay más mujeres. El curso académico con mayor porcentaje es el segundo con un 28,18\%, seguido del primer curso con un 23,64\%; y por último, el tercer curso con un $(21,82 \%)$.

Las características sociodemográficas de la muestra del personal bibliotecario se describen a través de las siguientes variables: el género, la escala profesional, la universidad, la biblioteca de trabajo. La muestra de éste se constituyó partiendo de la población de estudio de unos 808 bibliotecarios de las seis universidades madrileñas durante el curso académico 04/05 y 05/06, fecha de finalización del cuestionario quedando al final compuesta por unos 250 bibliotecarios.

En relación con el género del personal (Figuras 6, 7 y 8), predomina el género femenino $(66,80 \%)$, sobre el masculino $(33,20 \%)$, es decir, hay mayor presencia de mujeres que de hombres. El mayor número de encuestados los aportan las siguientes universidades: la Universidad Carlos III de Madrid (39,60\%), seguida de la Universidad Complutense de Madrid (36,00\%), la Universidad Autónoma de Madrid (9,6\%). El menor número, la Universidad Politécnica (3,20\%), por encima de ésta la Universidad Rey Juan Carlos (5,60\%) y la Universidad Pontificia de Comillas (6,00\%). La escala profesional de los encuestados mayoritariamente responde con un $43,6 \%$ a auxiliares, un $28,0 \%$ son ayudante, un 5,6\% facultativo, técnico un $4,4 \%$, becario un $7,6 \%$, y otros un $10,8 \%$.

FIGURA 6

Porcentaje de sujetos encuestados del personal bibliotecario por sexo

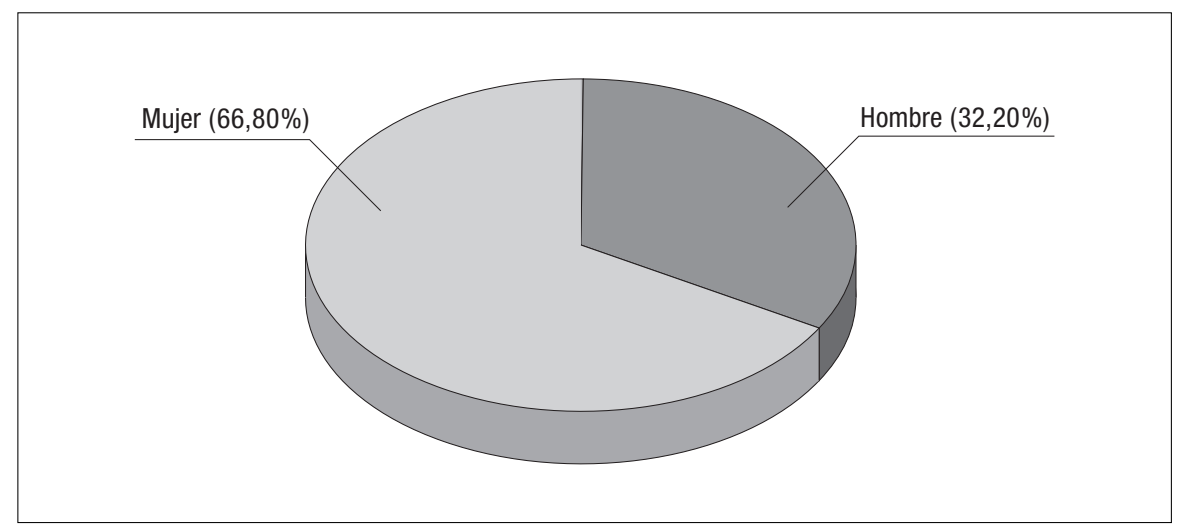




\section{FIGURA 7}

Personal encuestado por universidad

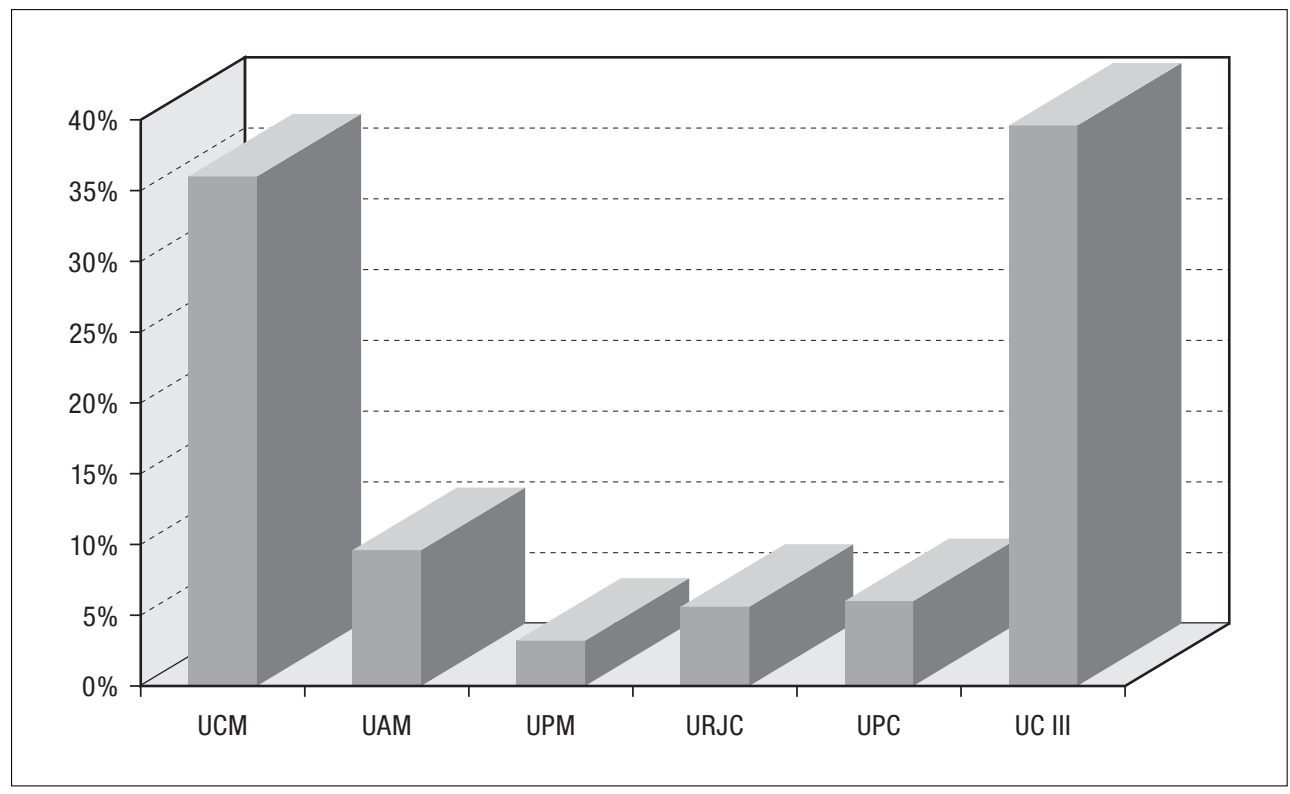

FIGURA 8

Porcentaje de la escala profesional del personal encuestado

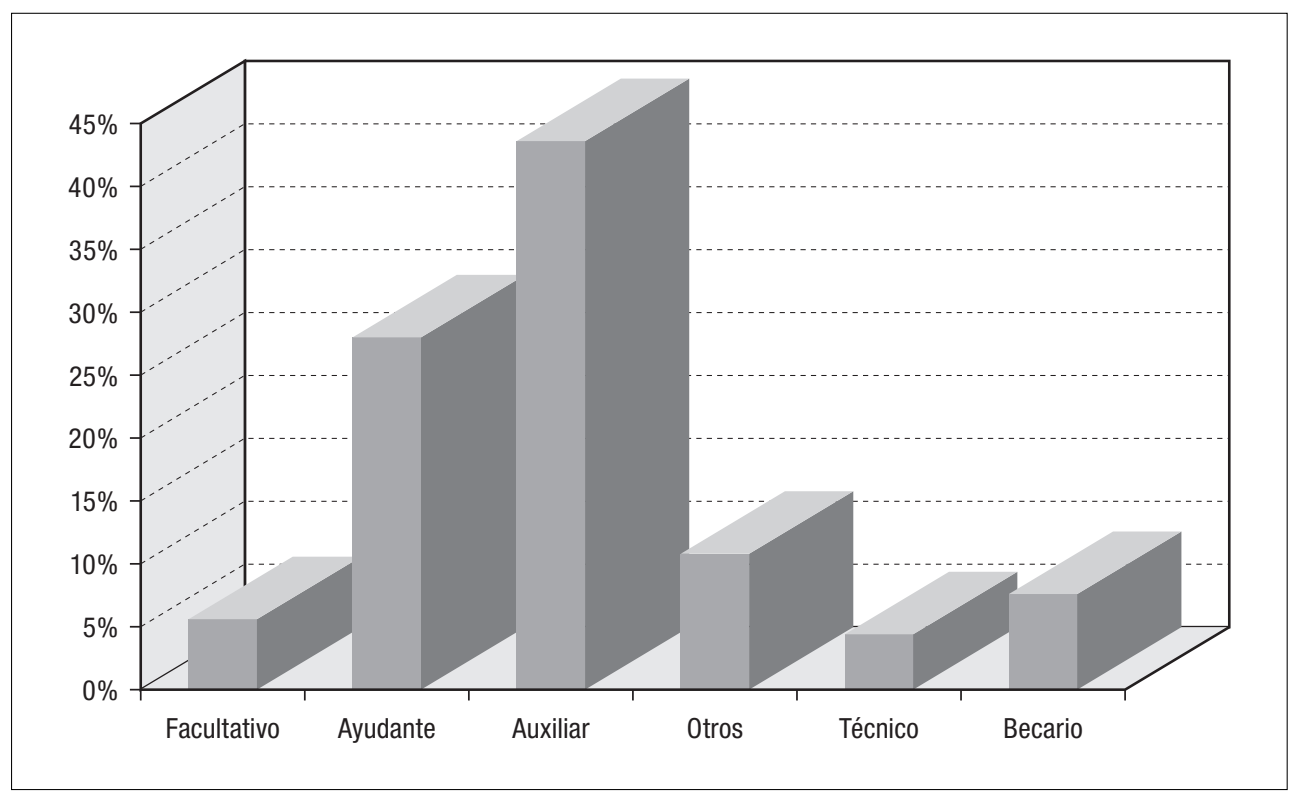


Las bibliotecas o centros de trabajo más frecuentes son las siguientes: con un mayor porcentaje nos encontramos las bibliotecas de Geografía e Historia (UCM), la E.U.I.T. de Telecomunicación (UPM), la E.U.I.T. de Minas (UPM), la F.C. de la Información (UCM), la F. Farmacia (UCM), el ICAI/ICADE (UPCO). Con un menor porcentaje de participación la E.T.S.I. Agrónomos (UPM), la F.C., de la Comunicación y Turismo (URJC), la de Humanidades (UAM).

\section{Resultados y discusión}

A continuación se presentan de forma sintética los resultados, agrupados por afinidad temática y de acuerdo con el criterio de relevancia estadística de ambos cuestionarios (el de usuarios con discapacidad y el de personal bibliotecario).

\section{- Usuarios con alguna discapacidad}

Los resultados relacionados con el cuestionario destinado a usuarios con alguna discapacidad tratan sobre sus hábitos, las infraestructuras, el personal, etc., entre otras cuestiones que se exponen a continuación.

El perfil mayoritario de los usuarios nos indica el uso del servicio de biblioteca con un $75 \%$. La frecuencia con un mayor porcentaje es una vez al mes, 34,9\%. El servicio más utilizado es el servicio de préstamo con un 51,2\%. Las razones de uso son un 44,2\% para devolver, renovar o tomar prestados libros. De acuerdo con estos elementos, el análisis bivariable indica que hay relación entre el tipo de discapacidad y el uso del servicio. También hay relación entre el tipo de discapacidad y la frecuencia de uso.

La opinión sobre las infraestructuras es positiva, la mayoría de los usuarios (62,7\%) opina que sí son adecuadas las instalaciones. Un 49,1\% responde que no dispone de señalizaciones perceptibles. El análisis bivariable nos indica que hay relación entre el tipo de discapacidad y la percepción de adecuación de las instalaciones.

La percepción del personal por los usuarios con alguna discapacidad indica que un $73,6 \%$ considera que el personal debería tener alguna formación específica. La valoración expresada de la atención del personal bibliotecario es considerada, ni muy valiosa, ni nada valiosa (34,5\%). El análisis bivariable nos indica que el tipo de discapacidad se relaciona con la consideración acerca de la formación que debería tener el bibliotecario.

En relación con los fondos, un $84,5 \%$ encuentra la bibliografía recomendada por el profesor. Un 57,3\% considera que sí dispone de materiales adaptados. Quienes consideran que no disponen de materiales adaptados o no lo saben, especificaron con un $72,0 \%$ que sería conveniente disponer de todas o varias de las opciones que se proponían (lector de pantalla, diccionarios ilustrados para sordos, etc). Aquí el análisis de relación entre variables, indica que el tipo de 
discapacidad influye en la percepción de la disponibilidad de materiales adaptados.

La valoración sobre los servicios web bibliotecarios y la facilidad de uso de los ordenadores para los estudiantes con discapacidad encuestados, nos indica con un $48,2 \%$ que son adecuados y con un $83,5 \%$ que sí deberían proporcionarse cursos de formación de usuarios, por lo que demuestran interés por aprender a emplear la biblioteca y mejorar sus habilidades. La relación entre variables nos sugiere que el tipo de discapacidad se relaciona con la percepción de la facilidad de uso de los servicios web, además de darse relación entre el tipo de discapacidad y la adecuación de los servicios web.

La recepción de información sobre algún servicio de apoyo a estudiantes con discapacidad nos lleva a decir que el mayor porcentaje de estudiantes encuestados afirma no haber recibido información (53,6\%). Para aquellos estudiantes con alguna discapacidad encuestados que sí han recibido información sobre algún servicio de apoyo, el canal más frecuente es el entorno académico (58,8\%). De todos los estudiantes encuestados un 60,9\% respondió no haber estado en ningún servicio de apoyo a estudiantes con discapacidad. De éstos, las razones argumentadas para no acudir son desconocimiento, 72,7\%. La relación entre variables nos indica que el tipo de discapacidad se relaciona con la recepción de información (las personas con una discapacidad física reciben más información que las personas con una discapacidad sensorial), así como se el tipo de discapacidad se relaciona con estancia en algún servicio de apoyo (acuden más las personas con una discapacidad física que las personas con una discapacidad sensorial).

\section{- Resultados sobre el personal}

Los resultados asociados al personal los presentamos también siguiendo los criterios anteriores para sintetizarlos, ’por lo que sólo expondremos los relacionados con la percepción y los hábitos, y los conocimientos-motivación.

La percepción del personal nos indica que un 62,8\% afirma conocer a algún usuario con alguna discapacidad. El tipo de discapacidad más frecuente es la motora/física con un $61,1 \%$. El personal percibe como servicio más empleado el préstamo con un $61,8 \%$. Con una frecuencia semanal de un 39,5\%. El personal afirma que se emplean todos los materiales (monografías, bases de datos, etc.), $47,8 \%$. Las razones percibidas por el personal indican que éste acude por todas las razones anteriores u otras $(34,4 \%)$, es decir, para consulta, préstamo, etc. El análisis bivariable en este caso nos indica que el conocimiento de usuarios con discapacidad se ve afectado por el centro de trabajo.

En cuanto a los conocimientos del personal sobre aspectos relacionados con la discapacidad y su motivación hacia esta problemática, el personal respondió, con un $64,4 \%$ tener conocimientos relacionados con la discapacidad. Un $92,8 \%$ de este personal no ha recibido formación para atender a este colectivo. Sin embargo, un 59,6\% estaría bastante interesado en recibirlos. 
Por otra parte, se pidió al personal encuestado que valorase los siguientes conocimientos: sobre tecnologías de asistencia, accesibilidad y usabilidad, sobre las discapacidades, sistemas alternativos de comunicación, o convenios de colaboración con entidades relacionadas con las personas con discapacidad, que habría de reunir el bibliotecario.

En el primero (debe conocer las tecnologías de asistencia), un 32,8\% aprueba totalmente.

El segundo (debe reunir conocimientos sobre accesibilidad y usabilidad), un 42, $4 \%$ aprueba totalmente.

El tercero (debe reunir conocimientos específicos sobre las discapacidades), un $44,4 \%$ se encuentra indeciso.

El cuarto (debe conocer los sistemas alternativos), el personal bibliotecario respondió con una actitud indecisa 33,6\%.

El quinto (debe conocer los convenios de colaboración de su biblioteca o centro con alguna asociación o entidad relacionada), generó una respuesta que en un $40,0 \%$ aprueban totalmente.

Por otro lado, sobre la recepción de información sobre las pautas WAI (Web Accesibility Initiative), un 82,8\% afirmó no haber recibido información. La relación entre variables nos permite decir que se da relación entre la escala profesional y la recepción de información sobre las pautas de accesibilidad WAI (reciben información mayormente facultativos y ayudantes), además de existir relación entre la biblioteca de trabajo y la recepción de información para atender a este colectivo (reciben formación en las bibliotecas de ciencias sociales y humanidades, antes que en ciencias e ingenierías).

\section{- Limitaciones}

La especificidad de la población no permite generalizar los resultados a lo que podríamos denominar "usuarios con discapacidad", ya que las características propias del ámbito universitario son distintas a la generalidad en la que se encuadraría esa población.

La utilización de la muestra nos ha aportado una serie de datos que la utilización del conjunto total de la población hubiese enriquecido. En sucesivas investigaciones se intentará mejorar esta cuestión.

La utilización de los cuestionarios ha sido una herramienta acertada para poder extraer la información, sin embargo, determinados datos e información no han podido sustraerse dadas las limitaciones propias de un cuestionario, algo que se tendrá en cuenta en posibles investigaciones posteriores.

El derecho a la intimidad y privacidad de datos dificulta la obtención de datos por parte de las instituciones encargados de gestionarlos (unidades o servicios de apoyo de las universidades), que se encuentran limitadas a la hora de colaborar. 


\section{Conclusiones}

Del marco teórico de la investigación se pueden extraer las siguientes, constantes que articulan la investigación que se está realizando sobre discapacidad y bibliotecas:

- Las discapacidades físicas y sensoriales son objeto de más interés en el campo de la investigación y en menor medida las psíquicas, corriente que se está abriendo en la actualidad.

- Los denominados servicios especiales, servicios bibliotecarios para personas con discapacidad, predominan en la de investigación frente a otros.

- Los centros que producen investigación son principalmente las bibliotecas públicas (asociada a las personas con discapacidad), seguidas de los centros escolares, universitarios y especiales.

- Las temáticas objeto del campo de la investigación sobre discapacidad y bibliotecas son diversas, van desde el acceso a la información, fuentes, accesibilidad y usabilidad, bases de datos, tecnologías de asistencia, formación de los bibliotecarios, estrategias bibliotecarias, alfabetización, etc.

Por otra parte dentro del marco teórico se constata la existencia de dos corrientes de acercamiento (activa o pasiva) a las personas con discapacidad y dos modelos conceptuales (modelo social y médico-curativo) con sus respectivas connotaciones. De estos dos modelos podemos decir que el modelo social es el que ha venido propiciando la creación de espacios de debate en el seno bibliotecario en relación con las personas con discapacidad. También dentro del marco teórico se ha podido constatar un creciente marco legal general que trata de responder a las demandas de las personas con discapacidad. No ocurre igual en el contexto legal bibliotecario dónde la visibilidad de éstos es menor.

\section{- Usuarios y personal}

Respecto a los usuarios con discapacidad se constata su presencia en la biblioteca universitaria, sus hábitos y uso con una frecuencia aceptable, el empleo de los distintos servicios por razones de uso, fundamentalmente. Tienen una buena opinión de las instalaciones, aunque consideran que no tienen señalizaciones perceptibles. Están de acuerdo en que el personal debería tener una formación específica para atenderles. Consideran la atención del personal bibliotecario ni muy valiosa/ ni nada valiosa en el mayor porcentaje. Perciben que disponen de materiales adaptados en la biblioteca; quienes no, sugieren los materiales que habría que tener. Piensan que los servicios bibliotecarios pueden mejorar.

Sobre el personal y la percepción del usuario con discapacidad, afirman conocerles y responden al resto de preguntas sobre sus hábitos. Lo que indica una 
sensibilidad del personal hacia estos usuarios, aunque no quiere decir que sean conscientes de otras cuestiones, necesidades, demandas, etc.

El personal encuestado, en su mayoría, no tiene conocimientos relacionados con la discapacidad, pero estaría interesado en recibir formación por parte de la biblioteca al respecto. La opinión acerca de los diferentes conocimientos que debería reunir el bibliotecario no es unánime para todos, pero es síntoma de la predisposición del personal en relación con la adquisición de conocimientos que mejoren su formación de cara a la atención a estas personas.

La mayoría del personal no sabe que tenga tecnologías de asistencia, lo que indica un desconocimiento de sus propios recursos y los de otros centros, máxime cuando es mediador, orientador entre el usuario y la biblioteca. Hay una parte que es consciente de la existencia de estos recursos y los indica (telelupa y otros). Admite que no ha recibido información sobre las pautas WAI y los pocos que la han recibido indican que el medio de comunicación es el entorno laboral. El personal tampoco sabe si sus servicios electrónicos son accesibles y cumplen dicha normativa.

Tampoco saben si existen servicios de apoyo universitarios para estudiantes con alguna discapacidad, o bien no los conocen. Lo que indica el desconocimiento de un medio que les puede facilitar la inclusión o la toma de medidas destinadas a mejorar el servicio mediante el asesoramiento de estos.

\section{- Biblioteca y discapacidad: cultura organizativa}

En relación con las bibliotecas y las personas con discapacidad se puede observar como todavía se han de afrontar los retos derivados del diseño para todos, se ha de fomentar una biblioteca o centro de recursos para el aprendizaje y la innovación abiertos. La inclusión de la discapacidad como un indicador de calidad a través de los cauces necesarios y de forma mensurable. Se constata la existencia de servicios bibliotecarios destinados a estudiantes con discapacidad, pero que no están extendidos en toda la geografía universitaria, tampoco hay una uniformidad en éstos.

Por lo tanto, sería aconsejable el fomento del conocimiento y uso de los servicios destinados por parte de la biblioteca para estas personas, así como buscar la normalización de criterios, características, etc., para que otras universidades los puedan implantar en sus bibliotecas.

Se observa la necesidad de mejorar la cultura organizativa de la biblioteca para favorecer la inclusión mejorando los flujos de información interna y externa. Flujos de comunicación que van desde los internos (biblioteca, universidad, comunidad universitaria), a los externos (biblioteca-otras instituciones-otras bibliotecas). Para ello se hace imprescindible la colaboración como una herramienta fundamental para lograr este objetivo, no sólo con otros servicios universitarios de atención a estudiantes con discapacidad u organizaciones relacionadas (asociaciones, etc.), sino con actuación de los diversos agentes implicados, personal 
bibliotecario, estudiantes, docentes, personal de administración y servicios, y demás miembros de la comunidad universitaria, cuya responsabilidad pasa por lograr una universidad de calidad.

\section{- Sensibilidad del bibliotecario e bipótesis}

Sería aconsejable fomentar el conocimiento del personal sobre estos usuarios y los recursos disponibles para ellos (servicios de apoyo universitarios, servicios bibliotecarios para estudiantes con discapacidad, medios para estos estudiantes, necesidades, etc.), actitudes positivas y un mejor conocimiento de la realidad. La recepción de cursos de formación, la creación de un espacio de diálogo constante con estos usuarios, para obtener un feedback que favorezca el uso de los servicios. Algunos aspectos a tener en consideración podrían ser la preparación de documentos para impresión, visualización y diseminación electrónica. Una ampliación de los plazos de préstamo, préstamo a domicilio (medida que se está aplicando en algunos centros como medida interna). Hemos de tener en cuenta que la movilidad académica y profesional que promueve el espacio europeo de educación superior hará que las estructuras sean más flexibles y adecuadas a las necesidades de los estudiantes.

En este contexto, el fomento de la percepción del bibliotecario como mediador en la instrucción sobre los recursos disponibles para los usuarios de la biblioteca ha de seguir reforzándose para favorecer su autonomía informativa, sobre todo, ante los usuarios con discapacidad, pues recordemos que la valoración que tenían era ni muy valiosa/ni nada valiosa.

Por otra parte, de acuerdo con las hipótesis planteadas, las opiniones expresadas por estos usuarios nos indican que usan el servicio de biblioteca y que la opinión sobre algunos aspectos es positiva. No obstante, son conscientes de que, en parte, los servicios de las bibliotecas universitarias han de mejorar en algunos aspectos para poder adecuarse a sus necesidades. Por lo que, en principio, nos permitiría aceptar nuestra primera hipótesis, es decir, la realidad de los servicios de las bibliotecas universitarias en la Comunidad de Madrid no está relacionada con las características de las personas con discapacidad. Continúan en la actualidad estando presentes barreras, que dificultan la inclusión de tipo arquitectónico, relacionadas con las tecnologías, de comunicación, accesibilidad, usabilidad y diseño para todos, aunque se esté trabajando en ello.

En relación con nuestra segunda hipótesis, en la Comunidad de Madrid, la realidad de los servicios bibliotecarios difiere entre personas con discapacidad y personal bibliotecario, podemos argumentar (tras la comparación de las opiniones en aquellos ítem similares), por ejemplo, la percepción del estudiante sobre los medios disponibles en la biblioteca, que consideraban que sí disponían, y la respuesta expresada por parte del personal que no sabe, o no conoce disponibilidad de tecnologías de asistencia (que vienen a ser los medios disponibles para los estudiantes) y que en su mayoría indicaban que no disponían de estas tec- 
nologías. Otro aspecto en que no coincide la percepción de ambos se da en relación con el tipo de discapacidad. El personal bibliotecario percibe más a los que tienen una discapacidad motora/física, seguidos de la auditiva y visual, mientras que el perfil del usuario con discapacidad nos indica una mayor presencia de personas con discapacidad visual (ciegas), seguidas de las que tienen discapacidad motora/física. Aunque en este sentido hemos de ser cautelosos, ya que las comparaciones no son todas adecuadas, aquí nos hemos limitado a argumentar aquellos aspectos relacionados en ambos cuestionarios.

A estas conclusiones se les pueden añadir las siguientes reflexiones y líneas de investigación como medio para lograr una mejora:

- Entornos inteligentes: la inclusión a través de las tecnologías, la domótica, la tecnología de redes etc., y podrían tener aplicación en las bibliotecas.

- La accesibilidad de los recursos a través de internet, la web semántica, la evaluación del hardware y software accesible, entornos virtuales y plataformas e-learning.

- Desarrollo, mejora, marketing y difusión por parte de la biblioteca universitaria de los servicios destinados a personas con discapacidad. La necesidad de proporcionar al personal cursos de formación. Ver como se puede mejorar la orientación de estos servicios para estas personas.

- En relación al personal, desarrollar el rol de mediador en la instrucción sobre el uso que se puede hacer de los recursos electrónicos en la enseñanza y aprendizaje electrónicos. Este puede servir de orientación para mejorar las políticas, normas y procedimientos para hacer accesibles dichos recursos.

- El análisis de la inclusión en el contexto laboral bibliotecario de las personas con discapacidad, o un estudio de las actitudes de este personal hacia la posible inclusión. La realización de programas de sensibilización y formación, analizando a posteriori sus posibles efectos dentro de la plantilla sería una línea interesante.

- En relación con la formación sería interesante promover la inserción de contenidos en las asignaturas y planes de estudio de biblioteconomía/documentación (transversalmente o directamente).

\section{Bibliografia}

ALFIN-EEES. [En línea], disponible en http://www.mariapinto.es/alfineees/AlfinEEES.htm [consulta: 26 de febrero de 2009].

Beverley, C. A.; Bath, P. A., y Barber, R. (2007): Can two established information models explain the information behaviour of visually impaired people seeking health and social care information. Journal of Documentation, 63 (1): 9-32.

Blandford, A., y Buchanan, G. (2003): Usability of digital libraries: a source of creative tensions with technical developments. TCLD Bulletin, Summer 2003. Disponible en 
http://www.ieee-tcdl.org/Bulletin/v1n1/blandford/blandford.html [consultado: $12 \mathrm{de}$ febrero de 2009].

Blog Alfin y discapacidad. [En línea], disponible en http://alfinenargentina.blogspot. com/2008/09/alfin-y-discapacidad.html [consulta: 26 de febrero de 2009].

Brazier, H. (2005): Charity, charges and chaos: the story of library services for visually impaired people in the UK. IFLA Council and General Conference, vol. 71. [En línea], disponible en http://www.ifla.org [consulta: 20 de febrero de 2009].

Byrne, A. (2005): Advancing library services for the blind in the global information society. IFLA Council and General Conference, vol. 71. [En línea], disponible en http:// www.ifla.org [consulta: 20 de febrero de 2009].

Carpenter, S. A. (1996): Accommodation to Persons with Disabilities: A Census of Ohio College and University Libraries. Katharine Sharp Review, 3. [En línea], disponible en http://www.ifla.org [consulta: 20 de febrero de 2009].

Cebrián de Miguel, M. D. (2004): Terminología de la discapacidad visual. The Translation Journal, 8 (1).

Centrum för Lättläst. (2000): Els representants de la lectura: un model que dóna oportunitats a persones amb limitacions per a la lectura. BiD: Biblioteconomía y Documentació, 4. [En línea], disponible en http://www.ub.eswww.ub.es/biblio/bid/04centru.htm [consultado: 20 de febrero de 2009].

Charoenpaed, M. (2000): Travelling books and the spirit of TamBoon. IFLA Council and General Conference, vol. 66.

Christensen, S. (2001): How We Work to Make the Web Speak. Computers in libraries, 21 (9).

Church, J.; Vaughan, J.; Starkweather, W., y Rankin, K. (2002): The Information Commons at Lied Library. Library bi tech, vol. 20 (1).

Cohen, U. (2000): Telebook. IFLA General Council and General Conference, nº 66. [En línea], disponible en http://www.ifla.org [consulta: 20 de marzo de 2009].

Coombs, N. (1999): New patrons: new changes. Library hi-tech, 17 (2).

Coombs, N. (2000): Enabling technologies: untangling your web. Library hi-tech, 18 (1), 93-96.

Coonin, B. (2002): Establishing accessibility for e-journals: a suggested approach. Library bi tech, vol. 20 (2).

Coonin, B. (2001): Enabling Scientists: Serving Sci-Tech Library Users with Disabilities. Issues in Science and Technology Librarianship, 32.

Desbuquois, C. (2002): L'accès à la lecture et à l'information des personnes handicapées visuelles: Réalités et perspectives. Bulletin des bibliothèques de France, 47 (6)

Dougnac, M. H. (2003): The reading experience of print-handicapped people: from Braille to new technologies. IFLA Council and General Conference, vol. 69. [En línea], disponible en http://www.ifla.org [consulta: 10 de febrero de 2009].

Draffan, E. A. B., y Corbett, R. (2002): Implementing a Web-accessible database. Electronic library, 19 (5).

Epp, M. A. (1999): Library services to Canadian college students with print disabilities. Library bi tech, 17 (2). 
España [Constitución Española de 1978]. Constitución Española. Aprobada por las Cortes el 31 de Octubre de 1978.

Evans, M. K. (2000): Serving the needs of visually impaired information seekers in UK public libraries. IFLA Council and General Conference, vol. 66. [En línea], disponible en http://www.ifla.org [consulta: 12 de febrero de 2009].

Forrest, M. E. S. (2005): Towards an accessible academic library: using the IFLA checklist. IFLA Council and General Conference, 71. [En línea], disponible en http://www.ifla. org [consulta: 12 de febrero de 2009].

Gallo León, J. P., y García Sáenz de Tejada, C. (2004): “Bibliotecas y personas discapacitadas: la experiencia de la Biblioteca Regional de Murcia". Foro Biblioteca y Sociedad. [En línea], disponible en http://www.anabad.org/admin/archivo/docdow.php?id=144 [consultado: 12 de febrero de 2009].

Griebel, R. (2000): Partnering Services between Public Libraries and Library Services for the Blind: A Canadian Experience. IFLA Council and General Conference, vol. 66. [En llínea], disponible en http://www.ifla.org [consulta: 15 de febrero de 2009].

Guerrero Quesada, D. J. (2005): MEDEA: Diseño de un sistema de información para la evaluación y la acreditación de titulaciones universitarias. [Tesis doctoral]. Granada: Universidad de Granada, 2005.

GUIADIS. [En línea], disponible en http://www.guiadis.es/ [consulta: 26 de febrero de 2009].

Gutiérrez y Restrepo, E. (2001): La accesibilidad en Internet: bibliotecas al servicio de tod@s. Educación y Biblioteca, vol. 15 (125): 109-111.

Halle e Eisenman. (2005): A Study of the Services and Equipment Provided to Physically Disabled Patrons at the University of North Carolina at Chapel Hill Libraries. A Master's Paper for the M.S. in L.S degree. November, 48 pages. Advisor: Diane Kelly.

Hassan Montero, Y., y Martín Fernández, F. J. (2004): Propuesta de adaptación de la metodología de diseño centrado en el usuario para el desarrollo de sitios web accesibles. Revista Española de Documentación Científica, 27 (3): 330-344.

Huidobro Verdera, M. (2001): Hacia la calidad en Internet. Educación y Biblioteca, vol. 15 (125): 98-108.

Kedem, K. (2000): "The "Disadvantaged" or "Special" Legislator Client: The Parliamentary Research Service's Challenge». IFLA Council and General Conference, 67. [En línea], disponible en http://www.ifla.org [consulta: 10 de febrero de 2009].

Cohen, U. (2000): Telebook. IFLA Council and General Conference, vol. 66. [En línea], disponible en http://www.ifla.org [consulta: 10 de febrero de 2009].

Ley 51/2003, de 2 de diciembre, de igualdad de oportunidades, no discriminación y accesibilidad universal de las personas con discapacidad.

Maldonado Inocencio, C. (1998): Hagamos las bibliotecas sin barreras arquitectónicas. Educación y Biblioteca, (87): 48-56.

Marqués i Soteras, A., y Sanahuja i Ballart, R. (1996): El mon de les discapacitats: bibliotheques, centres de documentacion i fons documentals. Item, 18: 132-152

Mayol, M. C. (1994) Serveis especials. Item, 14: 50-61.

Méndez Rodríguez, E. M. (2001): El acceso a la información un derecho de tod@as: bibliotecas y accesibilidad en la era de la información Web. Educación y Biblioteca, vol. 15 (125): 112-117. 
Millán Reyes, A. N. (2006): El reto de la Biblioteca Universitaria como recurso para la educación en el EEES: los servicios de apoyo, servicios especiales para las personas con discapacidad en las bibliotecas universitarias españolas. En: Adaptar la igualdad, normalizar la diversidad: II Congreso Nacional sobre Universidad y Discapacidad. XI Reunión del Real Patronato sobre Personas con Discapacidad. pp. 182-196. Madrid, 26-27 de octubre de 2006. Universidad Complutense.

Millán Reyes, A. N. (2008a): La Biblioteca Universitaria también al servicio de las personas con discapacidad: un estudio de caso. [Tesis doctoral]. Granada: Universidad de Granada.

Millán Reyes, A. N. (2008b): Bibliotecas Universitarias y personas con discapacidad: análisis del contexto legislativo en seis universidades madrileñas y andaluzas. $B A A B, 92-$ 93: 45-73.

O'neill, E. T. (1998): Characteristic of Web Accesible Information. Ifla Journal, vol. 24: 114-116.

Ortiz Macias, M. (2003): Discapacitados, desfavorecidos: la biblioteca, lugar de integración social. Educación y Biblioteca, vol. 15 (136), 99-102.

Pinto, M. (dir.); Millán, A. N.; Millán, M. J.; Fernández, A., y Iliberi, S. A. (2008a): PREDISCAN. [En línea], disponible en http://www.prediscan.com/ [consulta: 26 de febrero de 2009].

Pinto, M.; Sales, D., y Osorio, P. (2008b). Biblioteca universitaria, CRAI y alfabetización informacional. Gijón: Trea.

Pinto, M. (dir.); Millán, A. N.; Millán, M. J.; Gallego, M., y Iliberi, S. A. (2009): CREADIS. [En línea], disponible en http://www.creadiseees.org/cursos.html [consulta: 26 de febrero de 2009].

Pérez Pérez, J. R. (1998): Una biblioteca para discapacitados. Salamanca: Universidad Pontificia de Salamanca.

Romero Zúnica, R., et al. (1998): Biblioteca virtual: una forma de acceso a la información para personas con discapacidades físicas y sensoriales. Educación y Biblioteca (87): 76-78.

Saldaña Álvarez, L. (2008): La atención a usuarios con discapacidad. Una experiencia en la Biblioteca de la Universidad de Alcalá. Mi Biblioteca (14): 72-81.

Santos Chas, E. (2003): Recursos para la supresión de las barreras de comunicación de las personas sordas y sordociegas en el ámbito bibliotecario. Educación y Biblioteca (138): 78-80.

Suarez de la Llana, C. E. (1998): Las Nuevas Tecnologías de la Información y la Comunicación: posibilidades y perspectivas para el acceso a la información de las personas con discapacidad. Educación y Biblioteca (87): 43-47.

Teijo, C., y Barral, A. (2003): ¡Alerta! Nuevos servicios, nuevos usuarios. Educación y Biblioteca, vol. 15 (136): 93-94.

Térmens Graells, M.; Barrios Cerrejón, M.; Díaz Boladeras, M.; Guasch Murillo, D.; Ponsa Asensio, P., y Ribera Turró, M. (2008): Estudio de la accesibilidad de los documentos científicos en soporte digital. Revista Española de Documentación Científica, 31 (4): $552-572$. 